\title{
A New Class of Substituted Aryl Bis(oxazoline) Ligands for Highly Enantioselective Copper- catalyzed Asymmetric Aldol Addition of Dienolsilane to Pyruvate and Glyoxylate Esters
}

\author{
Julie Cong-Dung Le and Brian L. Pagenkopf* \\ The Department of Chemistry and Biochemistry, University of Texas at Austin, \\ Austin, TX 78712
}

pagenkopf@mail.utexas.edu

\section{Supporting Information}

\section{Contents}

Ligand synthesis S2

General procedure for pyruvate aldol:

\section{Experimental Section}

All reactions were run under an atmosphere of nitrogen or argon. Flasks were oven or flame-dried and allowed to cool in a desiccator prior to use. Solvents and reagents were purified by standard methods. ${ }^{1}$ Thin-layer chromatography (TLC) was performed on 60-F254 silica gel plates. The plates were visualized by staining with $\mathrm{I}_{2}$ on silica, $\mathrm{CAM},{ }^{2}$ or potassium permanganate. Enantiomeric excesses were determined by chiral HPLC with chiralpak AD and chiralcel OD columns: size: $250 \times 4.6 \mathrm{~mm}(\mathrm{~L} \times$ I.D), and UV detection $(245 \mathrm{~nm})$. Optical rotations were obtained at a wavelength of $589 \mathrm{~nm}$ (sodium D line) using a $1.0 \mathrm{dm}$ cell. Melting points are uncorrected. For ${ }^{1} \mathrm{H}$ NMR, chemical shifts are reported in parts per million (ppm) downfield from tetramethylsilane and are, in all cases, referenced to the residual

(1) Armarego, W. L. F.; Perrin, D. D. Purification of Laboratory Chemicals; 4th Ed.; Oxford: Butterworth-Heinemann, 1996.

(2) See footnote 50 in: Gao, Y.; Hanson, R.M.; Klunder, J.M.; Ko, S.Y.; Masamune, H.; Sharpless, K.B. J. Am. Chem. Soc. 1987, 109, 5765. 
proton resonance peaks: $\delta 7.24$ for $\mathrm{CHCl}_{3}$. The ${ }^{13} \mathrm{C}$ NMR chemical shifts were reported in ppm relative to the center peak of the solvent multiplet: $\delta 77.0$ (t) for $\mathrm{CDCl}_{3}$. HRMS (CI) were made with a VG analytical ZAB2-E instrument. Where appropriate, descriptions of signals include broad (b), apparent (app) and multiplet (m).

\section{Materials:}

Methyl pyruvate 1a, ethyl pyruvate 1b, ethyl 3-methyl-2-oxobutyrate 11, methyl benzoylformate 1e, and ethyl-4-nitrophenylglyoxalate $\mathbf{1 j}$ were purchased commercially. Benzyl pyruvate 1c and $t$-butyl pyruvate 1d were prepared by refluxing pyruvic acid and the corresponding alcohol in benzene and removing the water formed by azeotropic distillation. ${ }^{3} \alpha$-Keto esters $\mathbf{1 f}-\mathbf{i}$, and $\mathbf{1 k}$ were prepared by Grignard additions to diethyl oxalate. ${ }^{4}$ Glyoxalate $\mathbf{1 m}$ was prepared from ethyl pyruvate and benzaldehyde dimethyl acetal. ${ }^{5}$ Benzothiophene glyoxalate 1n was prepared according to a literature procedure. $^{6}$

\section{General procedure for preparing bisamides:}

To a solution of amino alcohol salt $(20 \mathrm{mmol})$ in $\mathrm{CH}_{2} \mathrm{Cl}_{2}(40 \mathrm{~mL})$ at $0{ }^{\circ} \mathrm{C}$ was added $\mathrm{Et}_{3} \mathrm{~N}(100 \mathrm{mmol})$. After $30 \mathrm{~min}$, a solution of dimethylmalonyl dichloride $(10 \mathrm{mmol})$ in $\mathrm{CH}_{2} \mathrm{Cl}_{2}(20 \mathrm{~mL})$ was added dropwise. The ice bath was removed and the thick light yellow suspension was stirred at rt for $1 \mathrm{~h}$. Following addition of $\mathrm{CH}_{2} \mathrm{Cl}_{2}(60 \mathrm{~mL})$, the mixture was washed with $\mathrm{HCl}(10 \mathrm{~mL}, 1 \mathrm{~N}), \mathrm{NaHCO}_{3}(20$ $\mathrm{mL}$, sat'd) and brine. The organics were dried $\left(\mathrm{Na}_{2} \mathrm{SO}_{4}\right)$, filtered through Celite and concentrated in vacuo to give a light yellow solid which was recrystallized from $\mathrm{Et}_{2} \mathrm{O} / \mathrm{EtOAc}$.

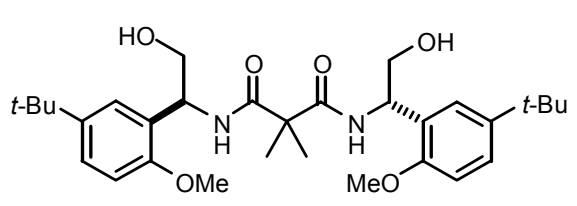

N,N'-Bis-[1-(5-tert-butyl-2-methoxy-phenyl)-2-hydroxyethyl]-2,2-dimethyl-malonamide (5a)

White solid (4.2 g, 78\%); $\mathrm{R}_{f} 0.20\left(10 \% \mathrm{MeOH} / \mathrm{CH}_{2} \mathrm{Cl}_{2}\right) ; \mathrm{mp} 134$ $-136{ }^{\circ} \mathrm{C} ;{ }^{1} \mathrm{H} \mathrm{NMR}\left(400 \mathrm{MHz}, \mathrm{CDCl}_{3}\right) \delta 7.75(\mathrm{~d}, J=8.8 \mathrm{~Hz}$, $4 \mathrm{H}), 7.28-7.22(\mathrm{~m}, 4 \mathrm{H}), 6.83(\mathrm{~d}, J=8.4 \mathrm{~Hz}, 2 \mathrm{H}), 5.31$ (ddd, $J=$ $13.2,9.2,6.8 \mathrm{~Hz}, 2 \mathrm{H}), 3.87(\mathrm{~s}, 6 \mathrm{H}), 3.79$ (d, $J=6.4 \mathrm{~Hz}, 4 \mathrm{H}), 1.46$ $(\mathrm{s}, 6 \mathrm{H}), 1.27(\mathrm{~s}, 18 \mathrm{H}) ;{ }^{13} \mathrm{C} \mathrm{NMR}\left(100 \mathrm{MHz}, \mathrm{CDCl}_{3}\right) \delta 173.8,154.8,143.6,126.1,125.4,125.2,110.5$, 65.5, 55.3, 54.6, 50.0, 34.0, 31.4, 23.4; HRMS m/z 543.3416 (calcd for $\mathrm{C}_{31} \mathrm{H}_{47} \mathrm{~N}_{2} \mathrm{O}_{6}[\mathrm{M}+\mathrm{H}]^{+}$, 543.3434).

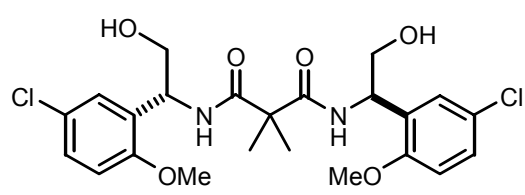

N,N'-Bis-[1-(5-chloro-2-methoxy-phenyl)-2-hydroxy-ethyl]2,2-dimethyl-malonamide (5b)

White solid (3.35 g, 75\%); $\mathrm{R}_{f} 0.23\left(10 \% \mathrm{MeOH} / \mathrm{CH}_{2} \mathrm{Cl}_{2}\right) ; \mathrm{mp} 158$ $-160{ }^{\circ} \mathrm{C} ;{ }^{1} \mathrm{H} \mathrm{NMR}(400 \mathrm{MHz}, \mathrm{DMSO}) \delta 7.86(\mathrm{~d}, J=8.0 \mathrm{~Hz}, 2 \mathrm{H})$, 7.24-7.20 (m, 4H), $6.96(\mathrm{~d}, J=8.8 \mathrm{~Hz}, 2 \mathrm{H}), 5.19$ (ddd, $J=13.2$, 9.2, $6.8 \mathrm{~Hz}, 2 \mathrm{H}), 4.93(\mathrm{dd}, J=6.8,6.8 \mathrm{~Hz}, 2 \mathrm{H}), 3.78(\mathrm{~s}, 12 \mathrm{H})$, 3.56-3.43 (m, 4H), $1.39(\mathrm{~s}, 6 \mathrm{H}) ;{ }^{13} \mathrm{C}$ NMR (100 MHz, DMSO) $\delta 182.7,165.0,140.5,137.3,136.8$, 134.0, 122.3, 72.5, 65.7, 60.1, 59.3, 33.6; HRMS $\mathrm{m} / z 499.3837$ (calcd for $\mathrm{C}_{23} \mathrm{H}_{29} \mathrm{Cl}_{2} \mathrm{~N}_{2} \mathrm{O}_{6}[\mathrm{M}+\mathrm{H}]^{+}$, 499.3829).

(3) Yamamoto, Y.; Maruyama, K.; Komatsu, T.; Ito, W. J. Org. Chem. 1986, 51, 886-891.

(4) (a) Marcitchie, J. A; Sicock, A.; Willis, C. L. Tetrahedron: Asymm. 1997, 8, 3895-3902. (b) Levy, A.; Rakowitz, A.; Mills, N. S. J. Org. Chem. 2003, 68, 3990-3998.

(5) Evans, D. A.; Johnson, J. S.; Olhava, E. J. J. Am. Chem. Soc. 2000, 122, 1635-1649.

(6) Kukelja, S.; Draheim, S. E.; Pfeil, J. L.; Cooper, R. D. G.; Graves, B. J.; Holmes, R. E.; Neel, D. A.; Huffman, G. W.; Webber, J. A.; Kinnick, M. D.; Vasileff, R. T.; Forster, B. J. J. Med. Chem. 1895, 28, 1886-1896. 


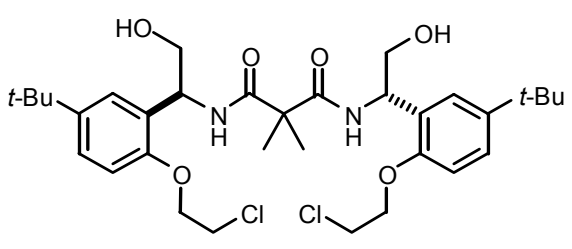

$N, N$ '-Bis-[1-(5-tert-butyl-2-chloroethoxy-phenyl)-2-hydroxyethyl]-2,2-dimethyl-malonamide (5c)

White solid (4.8 g, 75\%); $\mathrm{R}_{f} 0.27\left(10 \% \mathrm{MeOH} / \mathrm{CH}_{2} \mathrm{Cl}_{2}\right) ; \mathrm{mp} 142$ $-144{ }^{\circ} \mathrm{C} ;{ }^{1} \mathrm{H}$ NMR $(400 \mathrm{MHz}$, DMSO) $\delta 7.92(\mathrm{~d}, J=8.4 \mathrm{~Hz}$, $2 \mathrm{H}), 7.23(\mathrm{~d}, J=2.4 \mathrm{~Hz}, 2 \mathrm{H}), 7.15(\mathrm{dd}, J=8.4,2.4 \mathrm{~Hz}, 2 \mathrm{H}), 6.84$ (d, $J=8.8 \mathrm{~Hz}, 2 \mathrm{H}$ ), 5.23 (ddd, $J=13.2,9.2,6.8 \mathrm{~Hz}, 2 \mathrm{H}$ ), 4.85 (bs, 2H), 4.26-4.16 (m, 4H), 4.00-3.91 (m, 4H), 3.65-3.60 (m, $2 \mathrm{H}), 3.54-3.49(\mathrm{~m}, 2 \mathrm{H}), 1.37(\mathrm{~s}, 6 \mathrm{H}), 1.29(\mathrm{~s}, 18 \mathrm{H}) ;{ }^{13} \mathrm{C} \mathrm{NMR}\left(100 \mathrm{MHz}, \mathrm{CDCl}_{3}\right) \delta 173.8,153.5,144.2$, 126.4, 125.6, 125.4, 111.2, 67.9, 65.2, 54.6, 50.0, 42.1, 37.8, 34.0, 31.3, 23.5; HRMS $m / z$ 639.2984 (calcd for $\mathrm{C}_{33} \mathrm{H}_{49} \mathrm{Cl}_{2} \mathrm{~N}_{2} \mathrm{O}_{6}[\mathrm{M}+\mathrm{H}]^{+}, 639.2968$ ).

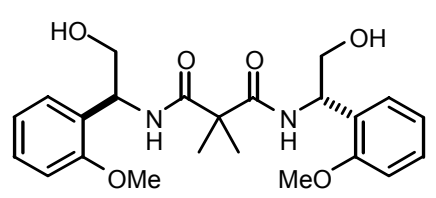

\section{$N, N$ '-Bis-[(2-methoxy-phenyl)-2-hydroxy-ethyl]-2,2-dimethyl- malonamide (5d)}

White solid (3.35 g, 78\%); $\mathrm{R}_{f} 0.22\left(10 \% \mathrm{MeOH} / \mathrm{CH}_{2} \mathrm{Cl}_{2}\right) ; \mathrm{mp} 135-137$ ${ }^{\circ} \mathrm{C}$; ${ }^{1} \mathrm{H}$ NMR $(300 \mathrm{MHz}, \mathrm{DMSO}) \delta 7.84(\mathrm{~d}, J=8.1 \mathrm{~Hz}, 2 \mathrm{H}), 7.21(\mathrm{~d}, J=$ $7.2 \mathrm{~Hz}, 2 \mathrm{H}), 7.16(\mathrm{~d}, J=7.8 \mathrm{~Hz}, 2 \mathrm{H}), 6.95(\mathrm{~d}, J=8.4 \mathrm{~Hz}, 2 \mathrm{H}), 6.83$ (dd, $J$ $=6.9,6.9 \mathrm{~Hz}, 2 \mathrm{H}), 5.27-5.21(\mathrm{~m}, 2 \mathrm{H}), 4.91(\mathrm{t}, J=5.9 \mathrm{~Hz}, 2 \mathrm{H}), 3.80(\mathrm{~s}$, $6 \mathrm{H}), 3.60-3.36(\mathrm{~m}, 4 \mathrm{H}), 1.38(\mathrm{~s}, 6 \mathrm{H}) ;{ }^{13} \mathrm{C} \mathrm{NMR}(75 \mathrm{MHz}, \mathrm{DMSO}) \delta 182.2,165.8,137.8,137.4,136.6$, $129.7,120.2,88.7,72.5,64.9,60.2,58.9,33.4$; HRMS $m / z 431.2165$ (calcd for $\mathrm{C}_{23} \mathrm{H}_{31} \mathrm{~N}_{2} \mathrm{O}_{6}[\mathrm{M}+\mathrm{H}]^{+}$, 431.2182).

\section{General procedure for preparing bis(oxazolines):}

To a solution of bisamide $(6.0 \mathrm{mmol})$ in $\mathrm{CH}_{2} \mathrm{Cl}_{2}(20 \mathrm{~mL})$ at $0{ }^{\circ} \mathrm{C}$ was added $\mathrm{Et}_{3} \mathrm{~N}(15 \mathrm{mmol})$ followed by a solution of $\mathrm{MsCl}(15 \mathrm{mmol})$ in $\mathrm{CH}_{2} \mathrm{Cl}_{2}(6.0 \mathrm{~mL})$. After stirring $12 \mathrm{~h}$ at $\mathrm{rt}$, the solution mixture was diluted with $\mathrm{CH}_{2} \mathrm{Cl}_{2}$ and poured into $\mathrm{NaHCO}_{3}(20 \mathrm{~mL}$, sat'd). The organic layer was removed and the aqueous layer was extracted with $\mathrm{CH}_{2} \mathrm{Cl}_{2}(3 \times 20 \mathrm{~mL})$. The combined extracts were washed with brine, dried $\left(\mathrm{Na}_{2} \mathrm{SO}_{4}\right)$, filtered through Celite and concentrated in vacuo to give a light yellow oil which was then dissolved in $\mathrm{MeOH}(10 \mathrm{~mL})$ and $\mathrm{KOH}$ powder $(15 \mathrm{mmol})$ was added. The suspension was heated at reflux for $2 \mathrm{~h}$. After cooling to rt, $\mathrm{H}_{2} \mathrm{O}(20 \mathrm{~mL})$ was added and the mixture was extracted with $\mathrm{CH}_{2} \mathrm{Cl}_{2}$ $(3 \times 10 \mathrm{~mL})$. The combined extracts were washed with brine, dried $\left(\mathrm{Na}_{2} \mathrm{SO}_{4}\right)$, filtered through Celite and concentrated in vacuo to give a light yellow residue which further purified by flash chromatography.

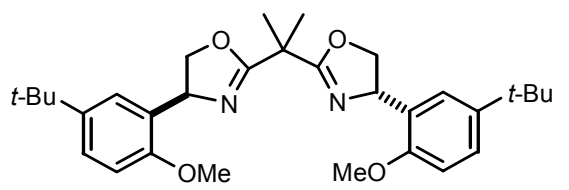

$(S, S)$-2,2'-Isopropylidene phenyl)-2-oxazoline] (6a)

bis[4-(5-tert-butyl-2-methoxy-

White crystalline solid (2.6 g, 85\%); $\mathrm{R}_{f} \quad 0.50 \quad(50 \%$ EtOAc/hexanes); mp $97-99{ }^{\circ} \mathrm{C} ;{ }^{1} \mathrm{H}$ NMR $\left(400 \mathrm{MHz}, \mathrm{CDCl}_{3}\right) \delta$ $7.34(\mathrm{~d}, J=2.8 \mathrm{~Hz}, 2 \mathrm{H}), 7.23$ (dd, $J=9.0,2.8 \mathrm{~Hz}, 2 \mathrm{H}), 6.78(\mathrm{~d}, J$ $=11.6 \mathrm{~Hz}, 2 \mathrm{H}), 5.45(\mathrm{dd}, J=13.2,9.2 \mathrm{~Hz}, 2 \mathrm{H}), 4.65(\mathrm{dd}, J=13.6,10.8 \mathrm{~Hz}, 2 \mathrm{H}), 4.07(\mathrm{dd}, J=9.2,9.2$ $\mathrm{Hz}, 2 \mathrm{H}), 3.79$ (s, 6H), 1.72 (s, 6H), 1.27 (s, $18 \mathrm{H}) ;{ }^{13} \mathrm{C} \mathrm{NMR}\left(100 \mathrm{MHz}, \mathrm{CDCl}_{3}\right) \delta 170.2,154.0,143.0$, 130.2 , 124.6, 124.2, 109.5, 75.0, 64.2, 55.2, 38.9, 34.1, 31.4, 24.7; HRMS m/z 507.3232 (calcd for $\mathrm{C}_{31} \mathrm{H}_{43} \mathrm{~N}_{2} \mathrm{O}_{4}[\mathrm{M}+\mathrm{H}]^{+}$, 507.3223).

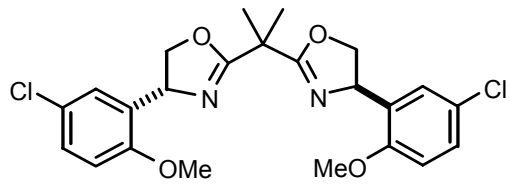

$(R, R)$-2,2'-Isopropylidene oxayoline] (6b) 
White crystalline solid (2.4 g, 86\%); $\mathrm{R}_{f} 0.40$ (50\% EtOAc/hexanes); mp $128-130{ }^{\circ} \mathrm{C} ;{ }^{1} \mathrm{H}$ NMR (300 $\left.\mathrm{MHz} \mathrm{CDCl}_{3}\right) \delta 7.29(\mathrm{~d}, J=2.7 \mathrm{~Hz}, 1 \mathrm{H}), 7.18(\mathrm{dd}, J=8.7,2.8 \mathrm{~Hz}, 1 \mathrm{H}), 6.75(\mathrm{~d}, J=8.7 \mathrm{~Hz}, 1 \mathrm{H}), 5.43$ $(\mathrm{dd}, J=9.9,7.5 \mathrm{~Hz}, 1 \mathrm{H}), 4.70(\mathrm{dd}, J=10.2,8.4 \mathrm{~Hz}, 1 \mathrm{H}), 3.99(\mathrm{dd}, J=8.1,8.1 \mathrm{~Hz}, 1 \mathrm{H}), 3.80(\mathrm{~s}, 3 \mathrm{H})$, $1.70(\mathrm{~s}, 3 \mathrm{H}) ;{ }^{13} \mathrm{C}$ NMR $\left(75 \mathrm{MHz}, \mathrm{CDCl}_{3}\right) \delta 170.7,154.7,132.8,127.7,126.9,125.6,111.1,74.7,63.7$, 55.5, 39.0, 24.4; HRMS $m / z$ 463.1168 (calcd for $\mathrm{C}_{23} \mathrm{H}_{25} \mathrm{Cl}_{2} \mathrm{~N}_{2} \mathrm{O}_{4}[\mathrm{M}+\mathrm{H}]^{+}, 463.1191$ ).

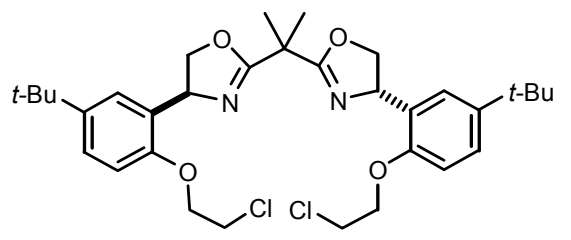

$(S, S)-2,2$ '-Isopropylidene phenyl)-2-oxazoline] (6c)

bis[4-(5-tert-butyl-2-chloroethoxySlightly yellow viscous oil (3.1 g, 85\%); $\mathrm{R}_{f} 0.55 \quad(50 \%$ EtOAc/hexanes); ${ }^{1} \mathrm{H}$ NMR (400 MHz, $\left.\mathrm{CDCl}_{3}\right) \delta 7.34(\mathrm{~d}, J=2.8$ $\mathrm{Hz}, 2 \mathrm{H}), 7.23$ (dd, $J=9.0,2.8 \mathrm{~Hz}, 2 \mathrm{H}), 6.78(\mathrm{~d}, J=11.6 \mathrm{~Hz}, 2 \mathrm{H})$, $5.45(\mathrm{dd}, J=13.2,9.2 \mathrm{~Hz} 2 \mathrm{H}), 4.65(\mathrm{dd}, J=13.6,10.8 \mathrm{~Hz}, 2 \mathrm{H}), 4.07$ (dd, $J=9.2,9.2 \mathrm{~Hz}, 2 \mathrm{H}), 3.79$ (s, $6 \mathrm{H}), 1.72(\mathrm{~s}, 6 \mathrm{H}), 1.27(\mathrm{~s}, 18 \mathrm{H}) ;{ }^{13} \mathrm{C} \mathrm{NMR}\left(100 \mathrm{MHz}, \mathrm{CDCl}_{3}\right) \delta 170.4,152.3,143.9,130.8,124.5$, 124.2, 110.4, 75.3, 67.9, 63.9, 42.2, 39.0, 34.2, 31.4, 24.6; HRMS $m / z 603.2746$ (calcd for $\mathrm{C}_{33} \mathrm{H}_{45}$ $\left.\mathrm{Cl}_{2} \mathrm{~N}_{2} \mathrm{O}_{4}[\mathrm{M}+\mathrm{H}]^{+}, 603.2756\right)$.

$(S, S)-2,2$ '-isopropylidene bis[4-(2-methoxy-phenyl)-2-oxazoline] (6d) White crystalline solid (2.0 g,

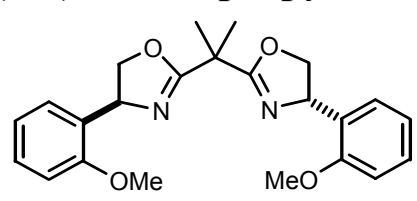
$85 \%) ; \mathrm{R}_{f} 0.45$ (50\% EtOAc/hexanes); mp $120-122{ }^{\circ} \mathrm{C} ;{ }^{1} \mathrm{H}$ NMR (400 $\left.\mathrm{MHz}, \mathrm{CDCl}_{3}\right) \delta 7.34(\mathrm{~d}, J=2.8 \mathrm{~Hz}, 2 \mathrm{H}), 7.25-7.21(\mathrm{~m}, 4 \mathrm{H}), 6.93(\mathrm{ddd}, J=$ $0.8,0.8,7.6 \mathrm{~Hz}, 2 \mathrm{H}), 6.85(\mathrm{~d}, J=8.4 \mathrm{~Hz}, 2 \mathrm{H}), 5.48(\mathrm{dd}, J=10.4,8.4 \mathrm{~Hz}$, $2 \mathrm{H}), 4.70(\mathrm{dd}, J=10.4,8.4 \mathrm{~Hz}, 2 \mathrm{H}), 4.02(\mathrm{dd}, J=8.0,8.0 \mathrm{~Hz}, 2 \mathrm{H}), 3.82$ $(\mathrm{s}, 6 \mathrm{H}), 1.69(\mathrm{~s}, 6 \mathrm{H}) ;{ }^{13} \mathrm{C} \mathrm{NMR}\left(100 \mathrm{MHz}, \mathrm{CDCl}_{3}\right) \delta 170.3,156.2,131.1$, 128.1, 126.9, 120.6, 109.9, 74.9, 64.1, 55.16 (d), 39.0, 24.5; HRMS m/z 395.1983 (calcd for $\mathrm{C}_{23} \mathrm{H}_{27} \mathrm{~N}_{2} \mathrm{O}_{4}$ $\left.[\mathrm{M}+\mathrm{H}]^{+}, 395.1971\right)$.

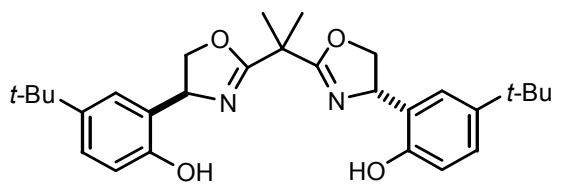

$(S, S)-2,2$ '-Isopropylidene phenyl)-2-oxazoline] (7a)

bis[4-(5-tert-butyl-2-hydroxy-

Off-white crystalline solid; $\mathrm{R}_{f} 0.70$ (50\% EtOAc/hexanes); mp $101-103{ }^{\circ} \mathrm{C} ;{ }^{1} \mathrm{H}$ NMR $\left(400 \mathrm{MHz}, \mathrm{CDCl}_{3}\right) \delta 9.26(\mathrm{bs}, 1 \mathrm{H}), 7.19$ (dd, $J=8.4,2.8 \mathrm{~Hz}, 2 \mathrm{H}), 6.81-6.80(\mathrm{~m}, 4 \mathrm{H}), 5.47$ (dd, $J=10.0$, $10.0 \mathrm{~Hz}, 2 \mathrm{H}), 4.89$ (dd, $J=10.4,8.0 \mathrm{~Hz}, 2 \mathrm{H}), 4.43$ (dd, $J=10.0$,

$8.0 \mathrm{~Hz}, 2 \mathrm{H}), 1.50(\mathrm{~s}, 6 \mathrm{H}), 1.27(\mathrm{~s}, 18 \mathrm{H}) ;{ }^{13} \mathrm{C} \mathrm{NMR}\left(100 \mathrm{MHz}, \mathrm{CDCl}_{3}\right) \delta 170.2,153.3,142.5,125.7$, 124.2, 122.9, 116.8, 74.4, 67.0, 92.2, 34.1, 31.5, 23.8; HRMS m/z 479.2905 (calcd for $\mathrm{C}_{29} \mathrm{H}_{39} \mathrm{~N}_{2} \mathrm{O}_{4}$ $\left.[\mathrm{M}+\mathrm{H}]^{+}, 479.2910\right)$.

\section{$[\mathbf{C u}-\mathrm{Box}] \mathbf{C l}_{2}$}

Bis(oxazoline) $(6,1.1 \mathrm{mmol})$ and $\mathrm{CuCl}_{2}(1.0 \mathrm{mmol})$ were mixed in a dry box and $\mathrm{CH}_{2} \mathrm{Cl}_{2}(4-6 \mathrm{~mL})$ was added. After being stirred at $\mathrm{rt}$ for $3.5 \mathrm{~h}$, the green clear solution was filtered through oven-dried Celite pad via cannula under Argon. The solvent was removed in vacuo to give a light green powder which was stored in a dry box.

\section{General procedure for pyruvate aldol:}

The copper catalyst ( $5 \mathrm{~mol} \%$ ) was added to a test tube in a dry box, the tube was sealed with a rubber septum and THF $(1.0 \mathrm{~mL})$ was added. After the mixture was stirred for $5 \mathrm{~min}$ at the indicated temperature, a solution of pyruvate $(0.5 \mathrm{mmol})$ in THF $(0.5 \mathrm{~mL})$ was added. After $30 \mathrm{~min}$, a solution of dienolate $(0.55 \mathrm{mmol})$ in THF $(0.5 \mathrm{~mL})$ was added dropwise. After $12-18 \mathrm{~h}$ at the indicated 
temperature, trifluoroacetic acid (TFA, $0.10 \mathrm{~mL}, 0.75 \mathrm{mmol})$ was added followed by $\mathrm{H}_{2} \mathrm{O}(0.5 \mathrm{~mL})$. The mixture was allowed to warm to $\mathrm{rt}$ and after $30 \mathrm{~min}$, EtOAc $(15 \mathrm{~mL})$ was added. The solution was washed with $\mathrm{NaHCO}_{3}\left(5 \mathrm{~mL}\right.$, sat'd) and brine $(5 \mathrm{~mL})$, dried $\left(\mathrm{Na}_{2} \mathrm{SO}_{4}\right)$ and filtered through Celite. Solvents were removed in vacuo, and the residue was purified by flash chromatography on silica gel with $20 \%$ hexanes/EtOAc to give the desired product as a colorless oil or crystalline solid $(67-91 \%$ yield).

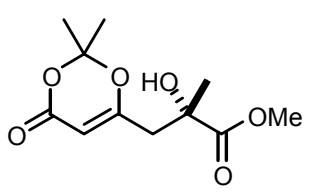

(S)-3-(2,2-Dimethyl-6-oxo-6H-[1,3]dioxin-4-yl)-2-hydroxy-2-methylpropionic acid methyl ester (3a)

Colorless oil (99 mg, 81\%); $\mathrm{R}_{f} 0.24$ (50\% EtOAc/hexanes); $[\alpha]^{26}{ }_{\mathrm{D}}+16.6(c 3.1$, $\left.\mathrm{CHCl}_{3}\right)$; IR $\left(\mathrm{CH}_{2} \mathrm{Cl}_{2}\right) 3446,1735,1632 \mathrm{~cm}^{-1} ;{ }^{1} \mathrm{H}$ NMR $\left(400 \mathrm{MHz}, \mathrm{CDCl}_{3}\right) \delta$ $5.28(\mathrm{~s}, 1 \mathrm{H}), 3.80(\mathrm{~s}, 3 \mathrm{H}), 3.35(\mathrm{bs}, 1 \mathrm{H}), 2.83(\mathrm{~d}, J=14.8 \mathrm{~Hz}, 1 \mathrm{H}), 2.56(\mathrm{~d}, J=$ $14.8 \mathrm{~Hz}, 1 \mathrm{H}), 1.63(\mathrm{~s}, 6 \mathrm{H}), 1.46(\mathrm{~s}, 3 \mathrm{H}) ;{ }^{13} \mathrm{C} \mathrm{NMR}\left(100 \mathrm{MHz}, \mathrm{CDCl}_{3}\right) \delta 176.1,166.9,160.8,106.7$, 96.1, 72.8, 52.9, 43.5, 27.0, 25.8, 23.9; HRMS $m / z 245.1024$ (calcd for $\mathrm{C}_{11} \mathrm{H}_{17} \mathrm{O}_{6}[\mathrm{M}+\mathrm{H}]^{+}, 245.1025$ ); Chiral HPLC (Daicel Chiralpak AD) hexanes: $i-\mathrm{PrOH}=90: 10$; flow rate: $1.0 \mathrm{~mL} / \mathrm{min} ; \lambda_{\max }=245 \mathrm{~nm}$; $\tau_{\text {minor }}=13.2 \mathrm{~min}, \tau_{\text {major }}=17.2 \mathrm{~min}$.

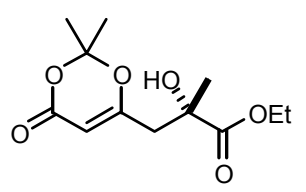

\section{(S)-3-(2,2-Dimethyl-6-oxo-6H-[1,3]dioxin-4-yl)-2-hydroxy-2-methyl-} propionic acid ethyl ester (3b)

Light yellow oil (97 mg, 75\%); $\mathrm{R}_{f} 0.26$ (50\% EtOAc/hexanes); $[\alpha]^{26}{ }_{\mathrm{D}}+33.6(c$ 3.1, $\left.\mathrm{CHCl}_{3}\right) ;{ }^{1} \mathrm{H} \mathrm{NMR}\left(300 \mathrm{MHz}, \mathrm{CDCl}_{3}\right) \delta 5.29(\mathrm{~s}, 1 \mathrm{H}), 4.32-4.19(\mathrm{~m}, 2 \mathrm{H})$, $3.38(\mathrm{bs}, 1 \mathrm{H}), 2.83(\mathrm{~d}, J=14.8 \mathrm{~Hz}, 1 \mathrm{H}), 2.56(\mathrm{~d}, J=14.8 \mathrm{~Hz}, 1 \mathrm{H}), 1.64(\mathrm{~s}, 3 \mathrm{H})$, $1.63(\mathrm{~s}, 3 \mathrm{H}), 1.45(\mathrm{~s}, 3 \mathrm{H}), 1.32(\mathrm{t}, J=7.2 \mathrm{~Hz}, 3 \mathrm{H}) ;{ }^{13} \mathrm{C} \mathrm{NMR}\left(100 \mathrm{MHz}, \mathrm{CDCl}_{3}\right) \delta 175.7,167.0,160.8$, 106.8, 96.2, 72.7, 62.4, 43.4, 27.1, 25.8, 24.0, 14.1; HRMS m/z 259.1180 (calcd for $\mathrm{C}_{12} \mathrm{H}_{19} \mathrm{O}_{6}[\mathrm{M}+\mathrm{H}]^{+}$, 259.1181); Chiral HPLC (Daicel Chiralpak AD) hexanes: $i$-PrOH $=90: 10$; flow rate: $1.0 \mathrm{~mL} / \mathrm{min}$; $\lambda_{\max }$ $=245 \mathrm{~nm} ; \tau_{\text {minor }}=11.6 \mathrm{~min}, \tau_{\text {major }}=15.1 \mathrm{~min}$.

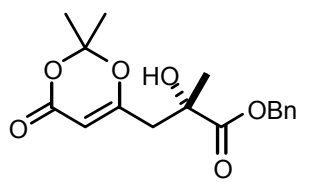

(S)-3-(2,2-Dimethyl-6-oxo-6H-[1,3]dioxin-4-yl)-2-hydroxy-2-methylpropionic acid benzyl ester (3c)

Light yellow oil (115 mg, 72\%); $\mathrm{R}_{f} 0.25$ (50\% EtOAc/hexanes); $[\alpha]^{26}{ }_{\mathrm{D}}+31.4(\mathrm{c}$ $\left.3.1, \mathrm{CHCl}_{3}\right) ;{ }^{1} \mathrm{H} \mathrm{NMR}\left(400 \mathrm{MHz}, \mathrm{CDCl}_{3}\right) \delta 7.42-7.35(\mathrm{~m}, 5 \mathrm{H}), 5.28(\mathrm{~s}, 1 \mathrm{H})$, $5.21(\mathrm{dd}, J=15.2,12.0 \mathrm{~Hz}, 2 \mathrm{H}), 3.34(\mathrm{bs}, 1 \mathrm{H}), 2.86(\mathrm{~d}, J=14.8 \mathrm{~Hz}, 1 \mathrm{H}), 2.58$ $(\mathrm{d}, J=14.8 \mathrm{~Hz}, 1 \mathrm{H}), 1.61(\mathrm{~s}, 3 \mathrm{H}), 1.55(\mathrm{~s}, 3 \mathrm{H}), 1.48(\mathrm{~s}, 3 \mathrm{H}) ;{ }^{13} \mathrm{C}$ NMR $\left(100 \mathrm{MHz}, \mathrm{CDCl}_{3}\right) \delta 175.5$, $166.8,160.7,134.7,128.7,128.2,106.7,96.2,72.8,67.9,43.3,27.1,25.7,23.9 ; \mathrm{HRMS} \mathrm{m} / z$ 321.1348 (calcd for $\mathrm{C}_{17} \mathrm{H}_{21} \mathrm{O}_{6}[\mathrm{M}+\mathrm{H}]^{+}, 321.1338$ ); Chiral HPLC (Daicel Chiralpak AD) hexanes:i-PrOH = 90:10; flow rate: $1.0 \mathrm{~mL} / \mathrm{min} ; \lambda_{\max }=245 \mathrm{~nm} ; \tau_{\text {minor }}=15.9 \mathrm{~min}, \tau_{\text {major }}=23.5 \mathrm{~min}$.

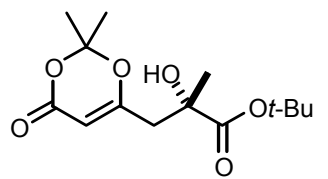

(S)-3-(2,2-Dimethyl-6-oxo-6H-[1,3]dioxin-4-yl)-2-hydroxy-2-methylpropionic acid tert-butyl ester (3d)

Colorless oil (100 mg, 70\%); $\mathrm{R}_{f} 0.30$ (50\% EtOAc/hexanes); mp $85-87{ }^{\circ} \mathrm{C}$; $[\alpha]^{26}+29.0\left(c 3.1, \mathrm{CHCl}_{3}\right) ;{ }^{1} \mathrm{H}$ NMR $\left(400 \mathrm{MHz}, \mathrm{CDCl}_{3}\right) \delta 5.31(\mathrm{~s}, 1 \mathrm{H}), 3.41$ (bs, $1 \mathrm{H}), 2.81(\mathrm{~d}, J=14.8 \mathrm{~Hz}, 1 \mathrm{H}), 2.53(\mathrm{~d}, J=14.8 \mathrm{~Hz}, 1 \mathrm{H}), 1.66(\mathrm{~s}, 3 \mathrm{H}), 1.65$ $(\mathrm{s}, 3 \mathrm{H}), 1.50(\mathrm{~s}, 9 \mathrm{H}), 1.42(\mathrm{~s}, 3 \mathrm{H}) ;{ }^{13} \mathrm{C}$ NMR $\left(100 \mathrm{MHz}, \mathrm{CDCl}_{3}\right) \delta 174.8,167.5,160.9,106.9,96.2,83.1$, 72.6, 43.3, 27.8, 27.4, 25.9, 24.0; HRMS $m / z 287.1490$ (calcd for $\mathrm{C}_{14} \mathrm{H}_{23} \mathrm{O}_{6}[\mathrm{M}+\mathrm{H}]^{+}, 287.1495$ ); Chiral 
HPLC (Daicel Chiralpak AD) hexanes: $i-\mathrm{PrOH}=90: 10$; flow rate: $1.0 \mathrm{~mL} / \mathrm{min} ; \lambda_{\max }=245 \mathrm{~nm}$; $\tau_{\operatorname{minor}}=$ $8.8 \mathrm{~min}, \tau_{\text {major }}=11.5 \mathrm{~min}$.

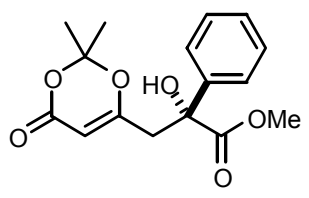

(S)-3-(2,2-Dimethyl-6-oxo-6H-[1,3]dioxin-4-yl)-2-hydroxy-2-phenylpropionic acid methyl ester (3e)

Clear crystalline solid (140 mg, 91\%); $\mathrm{R}_{f} 0.32$ (50\% EtOAc/hexanes); mp 110 $112{ }^{\circ} \mathrm{C} ;[\alpha]^{26}-9.5$ (c 3.1, $\left.\mathrm{CHCl}_{3}\right) ;{ }^{1} \mathrm{H}$ NMR (400 MHz, $\left.\mathrm{CDCl}_{3}\right) \delta$ 7.59-7.55 (m, $2 \mathrm{H}), 7.39-7.31(\mathrm{~m}, 3 \mathrm{H}), 5.32(\mathrm{~s}, 1 \mathrm{H}), 3.94(\mathrm{bs}, 1 \mathrm{H}), 3.82(\mathrm{~s}, 3 \mathrm{H}), 3.85(\mathrm{~d}, J=$ $14.8 \mathrm{~Hz}, 1 \mathrm{H}), 2.84(\mathrm{~d}, J=14.8 \mathrm{~Hz}, 1 \mathrm{H}), 1.64(\mathrm{~s}, 3 \mathrm{H}), 1.61(\mathrm{~s}, 3 \mathrm{H}) ;{ }^{13} \mathrm{C} \mathrm{NMR}\left(100 \mathrm{MHz}, \mathrm{CDCl}_{3}\right) \delta$ 174.4, 166.8, 160.8, 140.4, 128.4, 128.3, 125.1, 106.7, 96.5, 76.2, 53.4, 43.2, 25.7, 23.8; HRMS m/z 245.1024 (calcd for $\mathrm{C}_{11} \mathrm{H}_{17} \mathrm{O}_{6}[\mathrm{M}+\mathrm{H}]^{+}, 245.1025$ ); Chiral HPLC (Daicel Chiralcel OD) hexanes: $i-\mathrm{PrOH}$ $=70: 30$; flow rate: $1.0 \mathrm{~mL} / \mathrm{min} ; \lambda_{\max }=245 \mathrm{~nm} ; \tau_{\text {minor }}=7.0 \mathrm{~min}, \tau_{\text {major }}=24.4 \mathrm{~min}$.

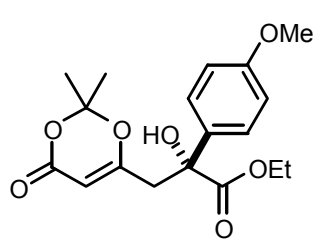

(S)-3-(2,2-Dimethyl-6-oxo-6H-[1,3]dioxin-4-yl)-2-hydroxy-2-(4-methoxyphenyl)-propionic acid ethyl ester (3f)

Light yellow oil (145 mg, 82\%); $\mathrm{R}_{f} 0.35$ (50\% EtOAc/hexanes); $[\alpha]^{26}{ }_{\mathrm{D}}-11.4(c$ $\left.3.1, \mathrm{CHCl}_{3}\right) ;{ }^{1} \mathrm{H} \mathrm{NMR}\left(400 \mathrm{MHz}, \mathrm{CDCl}_{3}\right) \delta 7.48(\mathrm{~d}, J=8.8 \mathrm{~Hz}, 2 \mathrm{H}), 6.88(\mathrm{~d}, J$ $=8.8 \mathrm{~Hz}, 2 \mathrm{H}), 5.32(\mathrm{~s}, 1 \mathrm{H}), 4.28-4.22(\mathrm{~m}, 2 \mathrm{H}), 3.93(\mathrm{bs}, 1 \mathrm{H}), 3.81(\mathrm{~s}, 3 \mathrm{H}), 3.32$

$(\mathrm{d}, J=14.8 \mathrm{~Hz}, 1 \mathrm{H}), 2.82(\mathrm{~d}, J=14.8 \mathrm{~Hz}, 1 \mathrm{H}), 1.65(\mathrm{~s}, 3 \mathrm{H}), 1.61(\mathrm{~s}, 3 \mathrm{H}), 1.31$ $(\mathrm{t}, J=7.2 \mathrm{~Hz}, 3 \mathrm{H}) ;{ }^{13} \mathrm{C} \mathrm{NMR}\left(100 \mathrm{MHz}, \mathrm{CDCl}_{3}\right) \delta 174.0,167.0,160.8,158.4,132.6,126.4,113.7$, 106.7, 96.4, 75.8, 62.8, 55.2, 43.2, 25.7, 23.8, 14.0; HRMS $m / z 351.1454$ (calcd for $\mathrm{C}_{18} \mathrm{H}_{23} \mathrm{O}_{7}[\mathrm{M}+\mathrm{H}]^{+}$, 351.1444); Chiral HPLC (Daicel Chiralcel OD) hexanes: $i-\mathrm{PrOH}=70: 30$; flow rate: $1.0 \mathrm{~mL} / \mathrm{min}$; $\lambda_{\max }=$ $245 \mathrm{~nm} ; \tau_{\text {minor }}=6.9 \mathrm{~min}, \tau_{\text {major }}=27.5 \mathrm{~min}$.

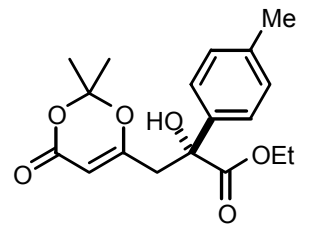

\section{(S)-3-(2,2-Dimethyl-6-0xo-6H-[1,3]dioxin-4-yl)-2-hydroxy-2-p-tolyl-} propionic acid ethyl ester (3g)

Light yellow oil (125 mg, 75\%); $\mathrm{R}_{f} 0.42$ (50\% EtOAc/hexanes); $[\alpha]^{26}{ }_{\mathrm{D}}-11.0(\mathrm{c}$ 2.3, $\left.\mathrm{CHCl}_{3}\right) ;{ }^{1} \mathrm{H} \mathrm{NMR}\left(400 \mathrm{MHz}, \mathrm{CDCl}_{3}\right) \delta 7.45(\mathrm{~d}, J=8.8 \mathrm{~Hz}, 2 \mathrm{H}), 7.16(\mathrm{~d}, J=$ $8.8 \mathrm{~Hz}, 2 \mathrm{H}), 5.31(\mathrm{~s}, 1 \mathrm{H}), 4.30-4.19(\mathrm{~m}, 2 \mathrm{H}), 3.94(\mathrm{bs}, 1 \mathrm{H}), 3.33(\mathrm{~d}, J=14.8 \mathrm{~Hz}$, $1 \mathrm{H}), 2.82(\mathrm{~d}, J=14.8 \mathrm{~Hz}, 1 \mathrm{H}), 2.34(\mathrm{~s}, 3 \mathrm{H}), 1.64(\mathrm{~s}, 3 \mathrm{H}), 1.60(\mathrm{~s}, 3 \mathrm{H}), 1.30(\mathrm{t}, J=$ $7.2 \mathrm{~Hz}, 3 \mathrm{H}) ;{ }^{13} \mathrm{C}$ NMR $\left(100 \mathrm{MHz}, \mathrm{CDCl}_{3}\right) \delta 174.0,167.0,160.9,138.1,137.7,129.1,125.1,106.8$, 96.4, 76.0, 62.8, 43.2, 25.8, 23.9, 20.9, 14.0; HRMS m/z 335.1505 (calcd for $\mathrm{C}_{18} \mathrm{H}_{23} \mathrm{O}_{6}[\mathrm{M}+\mathrm{H}]^{+}$, 335.1495); Chiral HPLC (Daicel Chiralcel OD) hexanes: $i-\mathrm{PrOH}=70: 30$; flow rate: $1.0 \mathrm{~mL} / \mathrm{min}$; $\lambda_{\max }=$ $245 \mathrm{~nm} ; \tau_{\text {minor }}=5.8 \mathrm{~min}, \tau_{\text {major }}=38.9 \mathrm{~min}$.

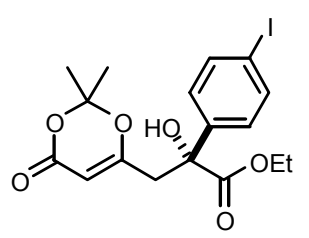

(S)-3-(2,2-Dimethyl-6-oxo-6H-[1,3]dioxin-4-yl)-2-hydroxy-2-(4-iodo-phenyl)propionic acid ethyl ester (3h)

Light yellow oil (200 mg, 90\%); $\mathrm{R}_{f} 0.41$ (50\% EtOAc/hexanes); $[\alpha]^{26}{ }_{\mathrm{D}}-22.0(\mathrm{c}$ 3.1, $\left.\mathrm{CHCl}_{3}\right)$; ${ }^{1} \mathrm{H}$ NMR $\left(400 \mathrm{MHz}, \mathrm{CDCl}_{3}\right) \delta 7.69(\mathrm{~d}, J=8.4 \mathrm{~Hz}, 2 \mathrm{H}), 7.33(\mathrm{~d}, J=$ $8.8 \mathrm{~Hz}, 2 \mathrm{H}), 5.32(\mathrm{~s}, 1 \mathrm{H}), 4.29-4.21(\mathrm{~m}, 2 \mathrm{H}), 4.00(\mathrm{bs}, 1 \mathrm{H}), 3.29(\mathrm{~d}, J=14.8 \mathrm{~Hz}$, $1 \mathrm{H}), 2.80(\mathrm{~d}, J=14.8 \mathrm{~Hz}, 1 \mathrm{H}), 1.63(\mathrm{~s}, 3 \mathrm{H}), 1.59(\mathrm{~s}, 3 \mathrm{H}), 1.31$ (t, $J=7.2 \mathrm{~Hz}, 3 \mathrm{H})$;

${ }^{13} \mathrm{C} \mathrm{NMR}\left(100 \mathrm{MHz}, \mathrm{CDCl}_{3}\right) \delta 173.5,166.4,160.7,140.4,137.5,127.3,106.8,96.7,94.3,75.9,63.2$, 43.1, 25.8, 23.9, 14.0; HRMS $m / z 447.0299$ (calcd for $\mathrm{C}_{17} \mathrm{H}_{20} \mathrm{IO}_{6}[\mathrm{M}+\mathrm{H}]^{+}$, 447.0305); Chiral HPLC (Daicel Chiralcel OD) hexanes:i-PrOH $=70: 30$; flow rate: $1.0 \mathrm{~mL} / \mathrm{min} ; \lambda_{\max }=245 \mathrm{~nm} ; \tau_{\operatorname{minor}}=6.6 \mathrm{~min}$, $\tau_{\text {major }}=37.4 \mathrm{~min}$. 


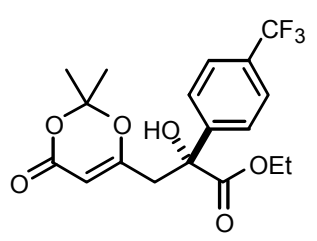

(S)-3-(2,2-Dimethyl-6-oxo-6H-[1,3]dioxin-4-yl)-2-hydroxy-2-(4trifluoromethyl-phenyl)-propionic acid ethyl ester (3i)

Light yellow oil (144 mg, 74\%); $\mathrm{R}_{f} 0.40$ (50\% EtOAc/hexanes); $[\alpha]^{26}{ }_{\mathrm{D}}+59.5(c$ $\left.3.1, \mathrm{CHCl}_{3}\right) ;{ }^{1} \mathrm{H} \mathrm{NMR}\left(400 \mathrm{MHz}, \mathrm{CDCl}_{3}\right) \delta 7.74(\mathrm{~d}, J=8.0 \mathrm{~Hz}, 2 \mathrm{H}), 7.63(\mathrm{~d}, J$ $=8.0 \mathrm{~Hz}, 2 \mathrm{H}), 5.33(\mathrm{~s}, 1 \mathrm{H}), 4.34-4.22(\mathrm{~m}, 2 \mathrm{H}), 4.01(\mathrm{bs}, 1 \mathrm{H}), 3.34(\mathrm{~d}, J=14.8$ $\mathrm{Hz}, 1 \mathrm{H}), 2.83$ (d, $J=14.8 \mathrm{~Hz}, 1 \mathrm{H}), 1.63$ (s, 3H), 1.39 (s, 3H), 1.33 (t, J=7.2 Hz, $3 \mathrm{H}) ;{ }^{13} \mathrm{C} \mathrm{NMR}\left(100 \mathrm{MHz}, \mathrm{CDCl}_{3}\right) \delta 173.2,166.3,160.7,144.5,125.9,125.4,125.3,106.9,96.7,75.9$, 63.4, 43.3, 31.5, 25.7, 23.9, 22.6, 14.0; HRMS $m / z 289.1217$ (calcd for $\mathrm{C}_{18} \mathrm{H}_{20} \mathrm{~F}_{3} \mathrm{O}_{6}[\mathrm{M}+\mathrm{H}]^{+}, 389.1212$ ); Chiral HPLC (Daicel Chiralcel OD) hexanes: $i-\mathrm{PrOH}=70: 30$; flow rate: $1.0 \mathrm{~mL} / \mathrm{min} ; \lambda_{\max }=245 \mathrm{~nm}$; $\tau_{\text {minor }}=5.4 \mathrm{~min}, \tau_{\text {major }}=8.5 \mathrm{~min}$.

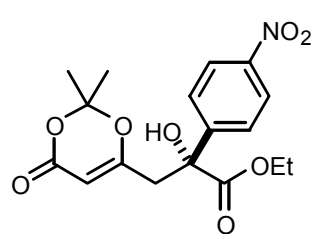

(S)-3-(2,2-Dimethyl-6-oxo-6H-[1,3]dioxin-4-yl)-2-hydroxy-2-(4-nitrophenyl)-propionic acid ethyl ester (3j)

Light yellow needles (145 mg, 77\%); $\mathrm{R}_{f} 0.29$ (50\% EtOAc/hexanes); mp 115 $117{ }^{\circ} \mathrm{C} ;[\alpha]^{26}{ }_{\mathrm{D}}-16.4\left(c\right.$ 3.1, $\left.\mathrm{CHCl}_{3}\right) ; \mathrm{IR}\left(\mathrm{CH}_{2} \mathrm{Cl}_{2}\right)$ 3467, 2979, 2935, 2371, $1741,1632 \mathrm{~cm}^{-1} ;{ }^{1} \mathrm{H}$ NMR $\left(300 \mathrm{MHz}, \mathrm{CDCl}_{3}\right) \delta 8.21(\mathrm{~d}, J=9.0 \mathrm{~Hz}, 2 \mathrm{H}), 7.80$ $(\mathrm{d}, J=9.0 \mathrm{~Hz}, 2 \mathrm{H}), 5.33(\mathrm{~s}, 1 \mathrm{H}), 4.34-4.24(\mathrm{~m}, 2 \mathrm{H}), 4.15(\mathrm{bs}, 1 \mathrm{H}), 3.34(\mathrm{~d}, J=14.8 \mathrm{~Hz}, 1 \mathrm{H}), 2.83(\mathrm{~d}, J$ $=14.8 \mathrm{~Hz}, 1 \mathrm{H}), 1.63(\mathrm{~s}, 3 \mathrm{H}), 1.59(\mathrm{~s}, 3 \mathrm{H}), 1.33(\mathrm{t}, J=7.2 \mathrm{~Hz}, 3 \mathrm{H}) ;{ }^{13} \mathrm{C} \mathrm{NMR}\left(75 \mathrm{MHz}, \mathrm{CDCl}_{3}\right) \delta 172.7$, $165.9,160.5,147.7,147.4,126.6,123.5,106.9,96.8,75.9,63.6,43.3,25.6,23.9$, 13.9; HRMS m/z 366.1183 (calcd for $\mathrm{C}_{17} \mathrm{H}_{20} \mathrm{NO}_{8}[\mathrm{M}+\mathrm{H}]^{+}, 366.1189$ ); Chiral HPLC (Daicel Chiralcel OD) hexanes:i$\mathrm{PrOH}=70: 30$; flow rate: $1.0 \mathrm{~mL} / \mathrm{min} ; \lambda_{\max }=245 \mathrm{~nm} ; \tau_{\operatorname{minor}}=7.9 \mathrm{~min}, \tau_{\text {major }}=10.5 \mathrm{~min}$.

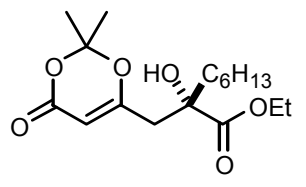

\section{2-(2,2-Dimethyl-6-0xo-6H-[1,3]dioxin-4-ylmethyl)-2-hydroxy-octanoic acid ethyl ester (3k)}

Light yellow oil (140mg, 85\%); $\mathrm{R}_{f} 0.39$ (50\% EtOAc/hexanes); $[\alpha]^{26}{ }_{\mathrm{D}}+19.7(c$ $\left.3.1, \mathrm{CHCl}_{3}\right) ;$ IR $\left(\mathrm{CH}_{2} \mathrm{Cl}_{2}\right) 3522,3017,2930,1741,1632 \mathrm{~cm}^{-1} ;{ }^{1} \mathrm{H}$ NMR (400 $\left.\mathrm{MHz}, \mathrm{CDCl}_{3}\right) \delta 5.27(\mathrm{~s}, 1 \mathrm{H}), 4.30-4.17(\mathrm{~m}, 2 \mathrm{H}), 3.38(\mathrm{bs}, 1 \mathrm{H}), 2.79(\mathrm{~d}, J=14.4$ $\mathrm{Hz}, 1 \mathrm{H}), 2.54(\mathrm{~d}, J=14.8 \mathrm{~Hz}, 1 \mathrm{H}), 1.77-1.58(\mathrm{~m}, 2 \mathrm{H}), 1.62(\mathrm{~s}, 3 \mathrm{H}), 1.61(\mathrm{~s}, 3 \mathrm{H}), 1.47-1.37(\mathrm{~m}, 1 \mathrm{H}), 1.30$ $(\mathrm{t}, J=7.2 \mathrm{~Hz}, 3 \mathrm{H}), 1.27-1.24(\mathrm{~m}, 6 \mathrm{H}), 1.08-1.00(\mathrm{~m}, 1 \mathrm{H}), 0.85(\mathrm{t}, J=6.8 \mathrm{~Hz}, 3 \mathrm{H}) ;{ }^{13} \mathrm{C} \mathrm{NMR}(100 \mathrm{MHz}$, $\left.\mathrm{CDCl}_{3}\right) \delta 175.3,167.2,160.8,106.7,96.1,75.5,62.2,42.8,39.8,31.5,29.0,25.9,23.9,22.9,22.4,14.0$; HRMS m/z 329.1959 (calcd for $\mathrm{C}_{17} \mathrm{H}_{29} \mathrm{O}_{6}[\mathrm{M}+\mathrm{H}]^{+}$, 329.1948); Chiral HPCL (Daicel Chiralpak AD) hexanes: $i-\mathrm{PrOH}=90: 10$; flow rate: $1.0 \mathrm{~mL} / \mathrm{min} ; \lambda_{\max }=245 \mathrm{~nm} ; \tau_{\operatorname{minor}}=9.4 \mathrm{~min}, \tau_{\mathrm{major}}=11.0 \mathrm{~min}$.

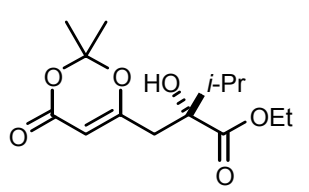

\section{(S)-2-(2,2-Dimethyl-6-0xo-6H-[1,3]dioxin-4-ylmethyl)-2-hydroxy-3-methyl-} butyric acid ethyl ester (3I)

Light yellow oil (107 mg, 75\%); $\mathrm{R}_{f} 0.28$ (50\% EtOAc/hexanes); $[\alpha]^{26}{ }_{\mathrm{D}}+17.6(c$ 2.6, $\left.\mathrm{CHCl}_{3}\right) ;{ }^{1} \mathrm{H} \mathrm{NMR}\left(400 \mathrm{MHz}, \mathrm{CDCl}_{3}\right) \delta 5.29(\mathrm{~s}, 1 \mathrm{H}), 4.33-4.17(\mathrm{~m}, 2 \mathrm{H}), 3.29$ (bs, 1H), $2.70(\mathrm{dd}, J=14.8,14.8 \mathrm{~Hz}, 2 \mathrm{H}), 1.99-1.92(\mathrm{~m}, 1 \mathrm{H}), 1.64(\mathrm{~s}, 3 \mathrm{H}), 1.63$ $(\mathrm{s}, 3 \mathrm{H}), 1.33(\mathrm{t}, J=7.2 \mathrm{~Hz}, 3 \mathrm{H}), 0.97(\mathrm{~d}, J=6.8 \mathrm{~Hz}, 3 \mathrm{H}), 0.85(\mathrm{~d}, J=6.4 \mathrm{~Hz}, 3 \mathrm{H}) ;{ }^{13} \mathrm{C} \mathrm{NMR}(100 \mathrm{MHz}$, $\left.\mathrm{CDCl}_{3}\right) \delta 175.4,167.8,160.9,106.7,96.1,77.9,62.2,40.5,35.8,25.8,23.9,17.0,16.0,14.2$; HRMS $m / z 287.1497$ (calcd for $\mathrm{C}_{14} \mathrm{H}_{23} \mathrm{O}_{6}[\mathrm{M}+\mathrm{H}]^{+}, 287.1495$ ); Chiral HPLC (Daicel Chiralpak AD) hexanes: $i-$ $\mathrm{PrOH}=90: 10$; flow rate: $1.0 \mathrm{~mL} / \mathrm{min} ; \lambda_{\max }=245 \mathrm{~nm} ; \tau_{\text {minor }}=8.7 \mathrm{~min}, \tau_{\text {major }}=11.5 \mathrm{~min}$. 


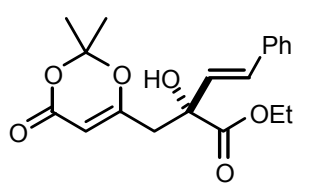

\section{2-(2,2-Dimethyl-6-oxo-6H-[1,3]dioxin-4-ylmethyl)-2-hydroxy-4-phenyl-but-} 3-enoic acid ethyl ester $(3 \mathrm{~m})$

Light yellow oil (140mg, 81\%); $\mathrm{R}_{f} 0.28$ (50\% EtOAc/hexanes); $[\alpha]^{26}-50.8(c$ 3.1, $\left.\mathrm{CHCl}_{3}\right) ;{ }^{1} \mathrm{H} \mathrm{NMR}\left(400 \mathrm{MHz}, \mathrm{CDCl}_{3}\right) \delta 7.38-7.25(\mathrm{~m}, 5 \mathrm{H}), 6.85(\mathrm{~d}, J=15.6$ $\mathrm{Hz}, 1 \mathrm{H}), 6.26(\mathrm{~d}, J=15.6 \mathrm{~Hz}, 1 \mathrm{H}), 5.34(\mathrm{~s}, 1 \mathrm{H}), 4.48(\mathrm{q}, J=7.2 \mathrm{~Hz}, 2 \mathrm{H}), 3.71$ (bs, 1H), $3.02(\mathrm{~d}, J=14.4 \mathrm{~Hz}, 1 \mathrm{H}), 2.65(\mathrm{~d}, J=14.4 \mathrm{~Hz}, 1 \mathrm{H}), 1.65(\mathrm{~s}, 3 \mathrm{H}), 1.62(\mathrm{~s}, 3 \mathrm{H}), 1.35(\mathrm{t}, J=7.2$ $\mathrm{Hz}, 3 \mathrm{H}) ;{ }^{13} \mathrm{C} \mathrm{NMR}\left(100 \mathrm{MHz}, \mathrm{CDCl}_{3}\right) \delta 173.6,166.6,160.8,135.7,130.8,128.6,128.4,128.2,126.7$, 106.8, 96.5, 75.4, 62.8, 42.8, 25.7, 24.0, 14.1; HRMS $m / z 347.1489$ (calcd for $\mathrm{C}_{19} \mathrm{H}_{22} \mathrm{O}_{6}[\mathrm{M}+\mathrm{H}]^{+}$, 347.1492); Chiral HPLC (Daicel Chiralpak AD) hexanes: $i-\mathrm{PrOH}=90: 10$; flow rate: $1.0 \mathrm{~mL} / \mathrm{min}$; $\lambda_{\max }$ $=245 \mathrm{~nm} ; \tau_{\text {minor }}=20.2 \mathrm{~min}, \tau_{\text {major }}=24.9 \mathrm{~min}$.

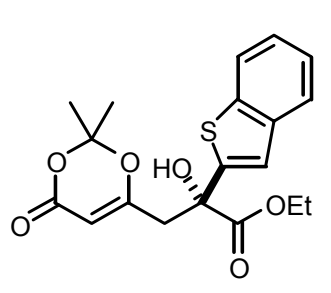

\section{2-Benzo[b]thiophen-2-yl-3(2,2-Dimethyl-6-oxo-6H-[1,3]dioxin-4-yl)- 2-} hydroxy-prpopionic acid ethyl ester (3n)

Clear needles (147 mg, 78\%); $\mathrm{R}_{f} 0.26$ (50\% EtOAc/hexanes); mp $118-120{ }^{\circ} \mathrm{C}$; $[\alpha]^{26}{ }_{\mathrm{D}}-24.7$ (c 2.5, $\left.\mathrm{CHCl}_{3}\right)$; IR $\left(\mathrm{CH}_{2} \mathrm{Cl}_{2}\right) 3462,3022,2990,1741,1638 \mathrm{~cm}^{-1}$; ${ }^{1} \mathrm{H}$ NMR $\left(400 \mathrm{MHz}, \mathrm{CDCl}_{3}\right) \delta 7.80(\mathrm{~d}, J=6.8,2.0 \mathrm{~Hz}, 1 \mathrm{H}), 7.71(\mathrm{~d}, J=6.8,2.0$ $\mathrm{Hz}, 1 \mathrm{H}), 7.36-7.32(\mathrm{~m}, 3 \mathrm{H}), 5.37(\mathrm{~s}, 1 \mathrm{H}), 4.35-4.30(\mathrm{~m}, 3 \mathrm{H}), 3.36(\mathrm{~d}, J=15.2$ $\mathrm{Hz}, 1 \mathrm{H}), 3.00(\mathrm{~d}, J=15.2 \mathrm{~Hz}, 1 \mathrm{H}), 1.65(\mathrm{~s}, 3 \mathrm{H}), 1.63(\mathrm{~s}, 3 \mathrm{H}), 1.38(\mathrm{t}, J=7.2$ $\mathrm{Hz}, 3 \mathrm{H}) ;{ }^{13} \mathrm{C} \mathrm{NMR}\left(100 \mathrm{MHz}, \mathrm{CDCl}_{3}\right) \delta 172.6,165.9,160.7,145.7,139.4,139.3,124.6,124.5,123.7$, 122.2, 121.2, 106.9, 96.7, 75.3, 63.4, 44.1, 25.7, 23.7, 14.0; HRMS $m / z$ 377.1054 (calcd for $\mathrm{C}_{19} \mathrm{H}_{21} \mathrm{O}_{6} \mathrm{~S}$ $\left.[\mathrm{M}+\mathrm{H}]^{+}, 377.1045\right)$; Chiral HPLC (Daicel Chiralcel OD) hexanes:i-PrOH $=50: 50$; flow rate: 1.0 $\mathrm{mL} / \mathrm{min} ; \lambda_{\max }=245 \mathrm{~nm} ; \tau_{\text {minor }}=6.8 \mathrm{~min}, \tau_{\text {major }}=52.2 \mathrm{~min}$. 